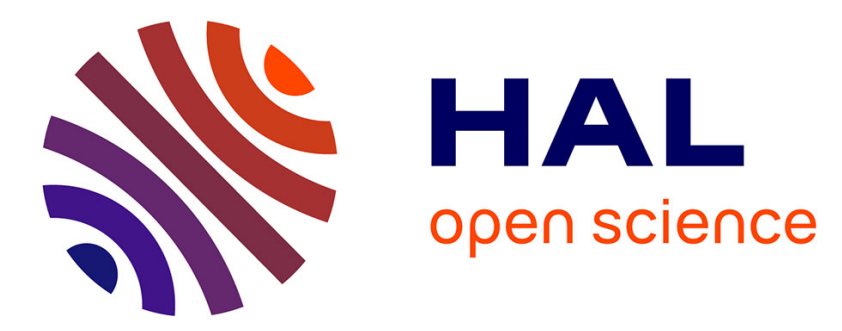

\title{
Plasmonic photocapacitance of self-assembled gold colloidal nanoparticle monolayers
}

H. Nesser, J. Grisolia, Adnen Mlayah, T. Alnasser, D. Lagarde, B. Viallet, L.

Ressier

\section{- To cite this version:}

H. Nesser, J. Grisolia, Adnen Mlayah, T. Alnasser, D. Lagarde, et al.. Plasmonic photocapacitance of self-assembled gold colloidal nanoparticle monolayers. Materials Today Nano, 2018, 4, pp.38 - 45. 10.1016/j.mtnano.2018.12.001 . hal-03486581

\section{HAL Id: hal-03486581 https://hal.science/hal-03486581}

Submitted on 20 Dec 2021

HAL is a multi-disciplinary open access archive for the deposit and dissemination of scientific research documents, whether they are published or not. The documents may come from teaching and research institutions in France or abroad, or from public or private research centers.
L'archive ouverte pluridisciplinaire HAL, est destinée au dépôt et à la diffusion de documents scientifiques de niveau recherche, publiés ou non, émanant des établissements d'enseignement et de recherche français ou étrangers, des laboratoires publics ou privés.

\section{(ㄷ)(1) $\$$}

Distributed under a Creative Commons Attribution - NonCommerciall 4.0 International 


\title{
Plasmonic photo-capacitance of self-assembled gold colloidal nanoparticle monolayers
}

\author{
H. Nesser ${ }^{\mathrm{a}}$, J. Grisolia ${ }^{\mathrm{a}}+$, A. Mlayah ${ }^{\mathrm{b}, \mathrm{c}}$, T. Alnasser ${ }^{\mathrm{a}}$, D. Lagarde $^{\mathrm{a}}$, B. Viallet ${ }^{\mathrm{a}}$ \\ and L. Ressier ${ }^{\mathrm{a}}$ \\ a. LPCNO, Université de Toulouse, INSA, CNRS, UPS, 135 avenue de Rangueil, Toulouse 31077, France. \\ b. CEMES, Université de Toulouse, CNRS, UPS, Toulouse, France \\ c. LAAS, Université de Toulouse, CNRS, UPS, Toulouse, France \\ †Corresponding author: jeremie.grisolia@insa-toulouse.fr
}

Electronic Supplementary Information (ESI) available: sample fabrication process, equivalent electrical circuit, capacitance versus temperature, DDA simulations, numerical simulations of photo-capacitance.

Keywords: photo-induced charge transport, surface plasmons, resistance, capacitance, gold nanoparticles, strain

\begin{abstract}
:
We report on the plasmo-electronic properties of self-assembled monolayers of gold colloidal nanoparticles (NPs) formed on a polyimide flexible substrate. The work is focused on the understanding of both resistance and capacitance of these NP assemblies. Their dependence on temperature, bias voltage, optical excitation and strain is investigated both experimentally and theoretically. Resistance and capacitance appear to bring complementary information on the charge transport properties of the NP assemblies as they exhibit opposite behaviors. Based on a nano-circuit junction model combined with numerical simulations, we were able to account for both the resistance and capacitance and their photo-induced modifications. The dependence of the capacitance on laser irradiation intensity and wavelength was measured and the role of the surface plasmon resonance was pointed out. We show that the plasmonic induced photocapacitance can reach $43 \%$ for a moderate irradiation intensity of $3.5 \mathrm{~W} / \mathrm{cm}^{2}$. Moreover, the NP assemblies, deposited on flexible substrate, were submitted to a uniaxial strain which allows for controlling the inter-particle distances and hence for probing the plasmo-electro-mechanical properties of the assembly. We found that the photo-capacitance gauge factor is enhanced by a factor 3 , by the plasmonic pumping of charges, compared to the one measured under dark conditions, in agreement with the calculations. The presented work thus paves the way to the emergence of a new class of plasmonic enhanced capacitance strain sensors.
\end{abstract}

\section{Introduction:}

The light-to-current transduction, via surface plasmon excitations and their decay into electrons and holes is the driving force of the emerging "plasmo-electronics" field which could provide a new class of opto-electronic devices [1]-[3]. It has been discovered that plasmonic excitations can influence macroscopic flows of charges and, conversely, that charging of metal 
nanoparticles (NPs) can impact their plasmonic response [1]. For instance, hot electrons generated by surface plasmons, may easily tunnel through interparticle distances larger than expected for thermalized electrons. Moreover, the strong plasmonic electromagnetic near-field localized in the gap regions between metal nanoparticles [4] may facilitate the electron tunneling, thus enhancing the current across NP assemblies. Interestingly, both positive and negative photoconductivities have been evidenced on a new class of nanoparticle-based materials [1].

However, the study of the plasmonic photo-conductivity is still in its infancy and efforts must be undertaken in order to unravel the complex physics of these nano-hybrid systems. Indeed, the conversion of light into current occurs through a chain process involving electronplasmon, electron-phonon and phonon-phonon interactions. These relaxation mechanisms still need a fundamental understanding in connection with size, shape and surface properties at the nanoscale. Energy relaxation through emission of phonons also leads to heat generation and to hyperthermia phenomenon which in turn give rise to thermo-induced currents. Moreover, charge transport through the composite medium formed by the metal nanoparticles and the surrounding molecules has different origins: quantum tunneling, variable range hopping, phonon assisted, excitation transfer assisted, carrier trapping/detrapping... Up to now, most of the published works, devoted to the charge transport in NP assemblies, have focused on the characterization and modeling of the total electrical resistance $R$ [5]-[8]. Very few studies were devoted to the total capacitance $C$ [9]-[10]. There is a major interest in combining both $R$ and $C$ measurements as both electrical characteristics contain complementary information on the nature of the charge transport mechanisms and on the plasmonic pumping of the photo-current.

In this work, we investigate the plasmonic induced charge transport in gold NP monolayered assemblies formed on a polyimide flexible substrate. We combine resistance and capacitance measurements under resonant excitation of the surface plasmons and applied mechanical strain in order to identify and characterize the photo-induced charge excitation and transport mechanisms. The effects of temperature, bias voltage, applied strain and optical excitation wavelength and intensity are investigated systematically. We found a clear correlation between the resistance and capacitance characteristics which we interpret using a strain-sensitive and photo-responsive circuit model which describes the electrical interconnection between the nanoparticles. To scale up to the macroscopic level we have performed numerical simulations of the charge transport throughout the whole NP assemblies. The numerical results and the experimental data are compared for both resistance and capacitance which allows gaining a deeper understanding of the photo-induced charge transport phenomenon in NP assemblies. 


\section{1 - Materials and methods:}

\section{1 - Sample preparation and characterization:}

Briefly, we first synthesized a colloidal dispersion of $14 \mathrm{~nm}$ spherical gold nanoparticles in toluene at a concentration of $2.10^{15} \mathrm{NPs} / \mathrm{ml}$ following the protocol of H. Hiramatsu et al.[11]. Second, $50 \mathrm{~nm}$ thick interdigitated gold electrodes were fabricated by laser photo-lithography (Dilase 650) on $125 \mu \mathrm{m}$ polyimide (Upilex) films using the lift-off method and the ion beam sputtering (IBS-E) technique. This results in a $1 \mathrm{~mm}^{2}$ area composed of 50 pairs of $5 \mu \mathrm{m}$ wide interdigitated electrodes (Figure 1a). Then, a layer of self-assembled gold NPs was formed on the whole area by the convective assembly technique described in [14] (Figure 1b). This method generates close-packed NP assembly with a long range as shown in Figure $1 b$ and $c$.

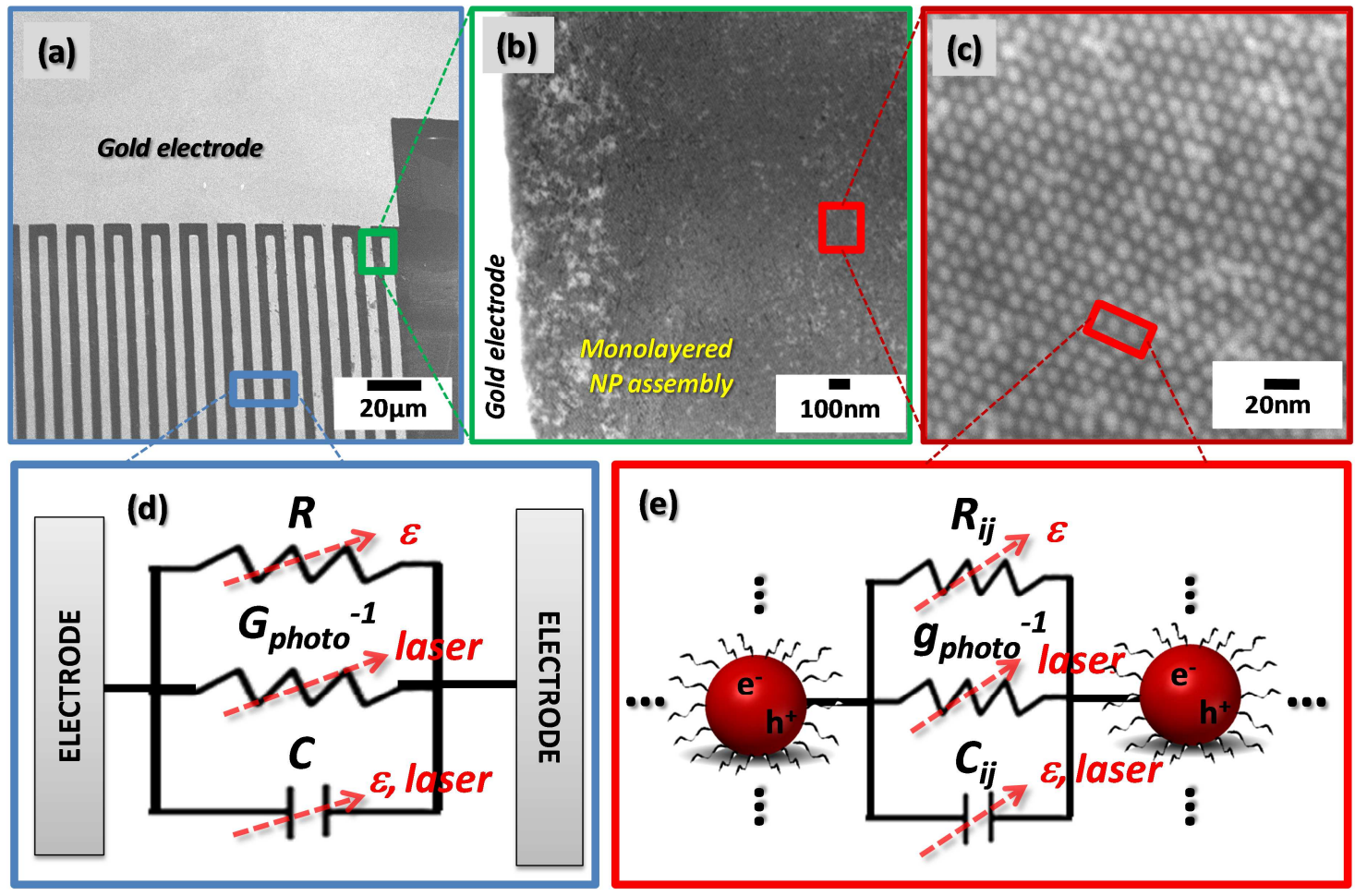

Figure 1 : (a) Scanning electron microscopy (SEM) the interdigited electrodes on top of which the NP assembly is formed, (b) and (c) high resolution SEM image of the active area showing the close-packed self-assembled monolayer of $14 \mathrm{~nm}$ gold nanoparticles (d) macroscopic model involving the total resistance $R$, capacitance $C$ and photo-conductance $G_{\text {photo }}(\mathrm{e})$ nano-circuit model of the electric inter-particle junction involving the tunnel resistance $\left(\boldsymbol{R}_{i j}\right)$, capacitance $\left(C_{i j}\right)$ and local photo-conductance $\left(g_{\text {photo }}\right)$. The red arrows indicate the parameters (strain $(\varepsilon)$ and laser excitations) to which these quantities are sensitive. 
Resistance and capacitance measurements were carried out using an impedance analyzer (Keysight E4990A) operating in the femto-Farads $(f F)$ capacitance range and in the 25 to $10 \mathrm{MHz}$ frequency range. A nanoparticle-free reference sample, with the same geometry, was fabricated and used for calibration, before each measurement, in order to get rid of the capacitance of the inter-digited electrodes and of the parasitic capacitances.

The optical excitation of the NP assembly was achieved using a CW laser operating either at $\lambda_{\text {inc }}=405,532$ or $640 \mathrm{~nm}$. The laser is coupled to an optical fiber and the output power is few tens of $\mathrm{mW}$ (near $40 \mathrm{~mW}$ ) depending on the excitation wavelength. The optical fiber is mounted in a Janis probe station which allows focusing the laser down to a spot size of $1 \mathrm{~mm}^{2}$ in order to illuminate the entire active area. The laser intensity was monitored using a diode driver. The maximum illumination intensity used in the experiments is $3.5 \mathrm{~W} / \mathrm{cm}^{2}$.

It is worthwhile to mention that the laser irradiation intensities used in this study are much weaker than the typical intensity $\left(10 \mathrm{~kW} / \mathrm{cm}^{2}\right)$ for photo-thermal induced heating in self-assembled $\mathrm{Au}$ nanoparticles. The photo-thermal effect may lead to melting of the nanoparticles and collapse of the assembly for laser irradiation above $40 \mathrm{~kW} / \mathrm{cm}^{2}$ [14].

Electro-mechanical experiments of the NP assemblies were conducted by applying a uniaxial stress to the flexible polyimide substrate using a traction machine (Deben Tensile Stage 200N). The applied force was increased from 0 to $70 \mathrm{~N}$ (by steps of $0.1 \%$ ).

\section{2 -Theory and numerical simulations:}

Calculations of the charge transport were performed assuming a monolayer of gold NPs arranged in a regular hexagonal lattice (Figure ESI 6) which mimics the actual arrangement of the NPs shown in Figure 1c. The NP diameter is $14 \mathrm{~nm}$ (i.e. the average size of the synthetized NPs) and the surface-to-surface distance is set to $1.2 \mathrm{~nm}$ (corresponding to the length of the $C_{12}$ molecules). Each nanoparticle, labeled $i$, is located at the node $\left(x_{i}, y_{i}\right)$ of the 2D network and defines, with the first neighbored particle $j$, an elementary parallel nano-circuit depicted in Figure 1e. The admittance $\chi_{i j}$ of this nano-circuit is given by:

$$
\text { (1) } \chi_{i j}=1 / R_{i j}\left(\varepsilon, V_{i j}, T\right)+g_{\text {photo }}\left(\lambda, I_{\text {laser }}\right)+j C_{i j} \omega .
$$

where $\omega$ is the angular frequency and $C_{i j}$ the inter-particle local capacitance.

The inter-particle resistance $R_{i j}$ is given by [7]:

$$
\text { (2) } \boldsymbol{R}_{i j}=\boldsymbol{k}_{R} \cdot e^{\xi\left(V_{i j}, T\right) l_{i j}(\varepsilon)}
$$

where $l_{i j}(\varepsilon)$ is the surface-to-surface interparticle distance which depends on the NP positions and hence on their change with applied strain $\varepsilon . k_{R}$ is a proportionality constant and the parameter $\xi$ accounts for the electron tunneling between nanoparticles [7]: 


$$
\text { (3) } \xi=\beta l-\ln \left(g\left(E_{c}, T, V_{i j}\right)\right) \text { with } g\left(E_{c}, T, V_{i j}\right)=\frac{1}{e V_{i j}}\left(\frac{-E_{C}-e V_{i j}}{1-e^{\left(E_{C}-e V_{i j}\right) / k_{B} T}}+\frac{E_{C}+e V_{i j}}{1-e^{\left(E_{C}+e V_{i j}\right) / k_{B} T}}\right)
$$

where the tunneling decay constant $\beta \sim 3 \mathrm{~nm}^{-1}$ has been estimated in previous works [12]. $\xi$ also depends on the Coulomb charging energy $E_{C}$, temperature $\mathrm{T}$ and voltage drop $V_{i j}$ between adjacent nanoparticles.

According to [13] and [9], the inter-particle capacitance $C_{i j}$ is given by

$$
\text { (4) } C_{\mathrm{ij}}=\pi \varepsilon_{0} \varepsilon_{r} d \cdot \ln \left(1+d / l_{i j}(\varepsilon)\right)
$$

where $d$ is the nanoparticle diameter, $\varepsilon_{0}$ is the vacuum permittivity, and $\varepsilon_{r}$ is the relative permittivity of the surrounding medium, i.e. of the organic ligand molecules in our case.

In order to introduce the inter-particle interaction in the admittance matrix, we assumed that all first neighbor nanoparticles located within a ring centered on the considered nanoparticle experience mutual capacitive influence.

In Equ. (1), $g_{\text {photo }}\left(\lambda, I_{\text {laser }}\right)$ accounts for the contribution of the optical excitation to the interparticle conductance, and depends only on the laser wavelength $\lambda$ (resonance effects) and intensity $I_{\text {laser }}$. The current flow, throughout the NPs assembly is computed by solving the matrix equation numerically [16]:

$$
\text { (5) }[V]=[Y]^{-1}[I]
$$

where $[V]$ and $[I]$ are respectively the voltage and the injected current vectors, $[Y]$ is the complex admittance matrix of which non-diagonal and diagonal terms are, respectively, $Y_{i j}=-\chi_{i j}(i \neq j)$ and $Y_{i i}$ $=\sum_{j} \chi_{i j}$; the latter is the total admittance connected to node $I$ [17]. The admittance is obtained according to $Y_{0}=V_{0} / I_{0}$ where $V_{0}$ is the last element of the voltage vector and $I_{0}$ is the injected current. Under dark conditions, the macroscopic resistance $R$ and capacitance $C$ of the whole NP assembly are determined using $R=1 / \operatorname{Re}\left(Y_{0}\right)$, and $C=\operatorname{Im}\left(Y_{0}\right) / \omega[17]$.

The numerical approach allows to take into account disorder effects due to fluctuations of the nanoparticle size and position, and to introduce a deformation (eventually non-uniform) of the NP network. For a perfectly ordered and strain-free NP network, it is possible to derive analytical expressions for the macroscopic total resistance $R$ and capacitance $C$ [7], [18]:

$$
\text { (6) } R=N_{R} \cdot R_{i j} \text { and } C=N_{C} \cdot C_{i j}
$$

where $N_{R}$ and $N_{C}$ are the numbers of series and parallel inter-particle junctions. The macroscopic resistance $R$ depends on temperature and bias voltage through the inter-particle resistance $R_{i j}$. The voltage drop between nanoparticles $V_{i j}$ can then be approximated by $V_{i j}=V_{b} / N_{j}$ where $V_{b}$ is the applied bias voltage and $N_{j}$ is the number of junctions between electrodes in order to scale up the model from nano to macro circuit. 
In the calculations, the optical excitation is treated at the macroscopic level: once the total resistance $R$ and capacitance $C$ are determined numerically, $G_{\text {photo }}$ is plugged into the parallel circuit as shown in Figure 1d. We adopt this approach for two main reasons. First, there have been very few theoretical works on the inter-particle photo-induced conductance and on the role of optical resonances [31]; the predicted properties still need to be compared to experiments. On the other hand, the available measurements of the photo-conductance were performed at the macroscopic level (i.e. on large NP assemblies), only. The photo-induced modification of the charge transport between two isolated nanoparticles requires further experimental efforts.

Mangold et al. [2], [15] and Gauvin et al. [14] showed that, for low laser irradiation (below $7 \mathrm{~kW} / \mathrm{cm}^{2}$ ), the photo-conductance increases linearly with increasing laser intensity due to the photothermal induced heating of the NP assemblies:

$$
\text { (7) } G_{\text {photo }}(p S) \approx k_{\text {photo }}(\lambda) * I_{\text {Laser }}\left(\frac{k W}{c m^{2}}\right)
$$

where the constant $k_{\text {photo }}(\lambda)$ accounts for the optical resonances and for the transduction of the electromagnetic energy into electric current. For dodecanethiol coated Au NPs, a $k_{\text {photo }} \sim 35.5$ $\mathrm{pS.cm} / \mathrm{kW}$ at plasmon resonance $\left(\boldsymbol{\lambda}_{\text {inc }} \mathbf{- 6 3 8} \mathbf{n m}\right)$ was found for optical excitation tuned to their plasmonic resonance [14].

\section{3- Results and Discussion:}

\section{1 - Resistance and Capacitance dependence on temperature and bias voltage:}

\subsubsection{Correlations between total resistance and total capacitance:}

We first investigate the charge transport through the NP assembly without any applied optical excitation or mechanical stress. Our aim is to point out the correlation between resistance and capacitance characteristics.

Figure 2a shows the temperature dependence of the total resistance $R$ and total capacitance $C$ :

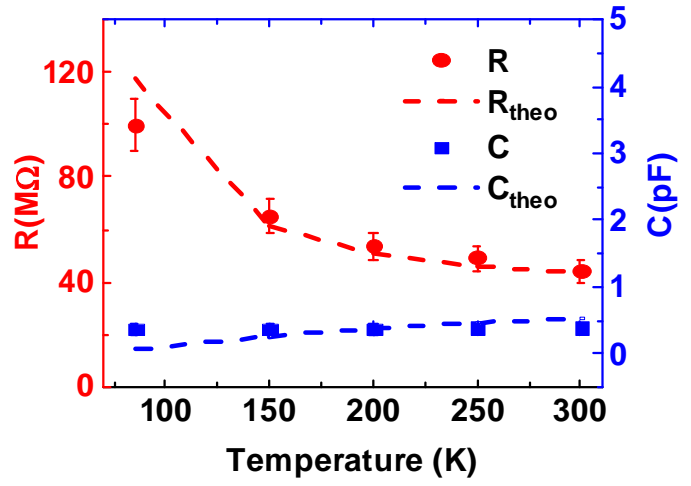

(a)

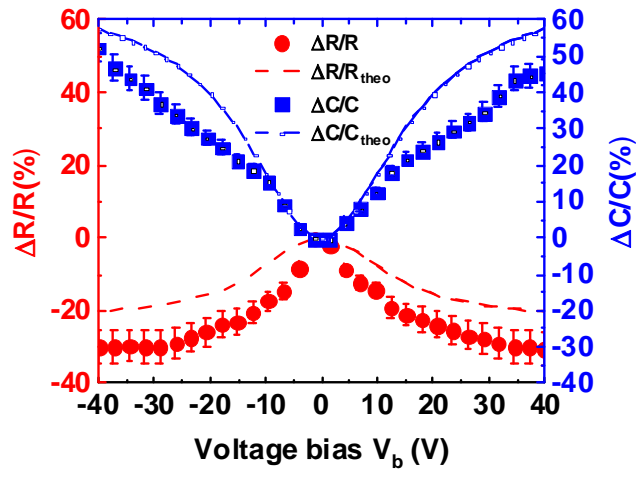

(b) 
Figure 2 : (a) Resistance and capacitance of the NP assembly versus temperature. The AC bias voltage is set to $100 \mathrm{mV}$ and $500 \mathrm{kHz}$ (b) Room temperature variations of the relative resistance $\Delta R / R$ and capacitance $\Delta C / C$ as a function of bias voltage. The dots and squares in (a) and (b) show the results of the measurements. The dashed lines are calculated curves using equations (2), (3), (6) and (8) and adjusted to the experimental data.

The total resistance $R$ is in the order of few hundreds of $\mathrm{M} \Omega$ and exhibits a non-metallic behavior, i.e. it decreases with increasing temperature which suggests that the surrounding molecules act as efficient inter-particle barriers against electron tunneling, as already reported in previous works [7], [18]. On the contrary, the capacitance increases with increasing temperature (see also Figure ESI 4) and reaches $350 \mathrm{fF}$ at room temperature (Figure 2a). It was suggested that the thermal expansion of the polyimide substrate could be responsible for the temperature variation of electrical properties (Yajadda et al. [19]). However, calculations showed that the substrate effect is negligible given the rather weak thermal expansion coefficient of polyimide $30-60 \times 10^{-6} \mathrm{~K}^{-1}$ [20].

Figure $2 \mathrm{~b}$ displays the variations, at room-temperature, of the relative resistance $\Delta R / R$ and relative capacitance $\Delta C / C$ as a function of bias voltage. $\Delta R / R$ is symmetric with respect to the sign of the bias voltage and decreases from 0 to $c . a .-30 \%$ at $+/-40 \mathrm{~V} . \Delta C / C$ is also symmetric with respect to the bias voltage and increases from 0 to c.a. $+45 \%$ at $+/-40 \mathrm{~V}$. As for the temperature dependence (Figure 2a), the change of $\Delta R / R$ and $\Delta C / C$ with bias voltage are opposite (Figure $2 \mathrm{~b}$ ). While the resistance properties of NP assemblies are fairly well understood [6], [18]-[26], to the best of our knowledge, so far, there has been no interpretation of the relationship between resistance and capacitance observed experimentally.

\subsubsection{Correlation between resistance and capacitance:}

The electrical properties of the NP assembly were analyzed using numerical simulations of the charge transport (presented in section 2) which allow deriving the macroscopic resistance $R$ and capacitance $C$ of the NP assemblies from the nano-circuit model (Figure 1e). The parallel circuit, formed by the macroscopic resistance $R$ and capacitance $C$ (Figure 1d) can be transformed into an equivalent circuit, and it can be shown the relation between the macroscopic capacitance and resistance is then given by [27], [28]:

$$
\text { (8) } C\left(T, V_{b}\right)=C_{\infty}+\frac{1}{\omega^{2} R\left(T, V_{b}\right)^{2} C_{\infty}}
$$

where $\omega$ is the angular frequency and $C_{\infty}$ is the high frequency total capacitance. The electrode resistance $r_{s}$, which is very small with respect to the total resistance of the NP assembly (few $\Omega$ compared to few M $\Omega$ ), can be included in the total resistance (see ESI Section II). 
Equ. (8) shows that $R$ and $C$ are inversely proportional and hence should vary in opposite directions as a function of temperature and bias voltage, in agreement with the experimental observations Figure 2. More precisely, Figure 2 presents a comparison between calculated and measured resistance and capacitance. The calculated curves (using Equ. (2), (3) combined to Equ. (6) and (8)) were adjusted to the measured ones using three fitting parameters: the number of junction $N_{j}$ between two electrodes, the charging energy $E_{c}$ and the total capacitance $C_{\infty}$. According to the geometrical characteristics of the NP assembly $(5 \mu \mathrm{m}$ length, $14 \mathrm{~nm}$ thick, $14 \mathrm{~nm}$ NP size, and $1.2 \mathrm{~nm}$ ligand length), the number of junctions is estimated to be c.a. $N_{j} \sim 300$. In the numerical simulations, this value is taken as an initial input parameter and can vary between 100 and 500 (junctions). After convergence, we obtain $N_{j}=305$ junctions, $E_{c}=20 \mathrm{meV}$ and $C_{\infty}=10 \mathrm{fF}$ [9]. It is worth mentioning that the fitting procedure was performed simultaneously using all experimental data, i.e. using the variations of both $R$ and $C$ as a function of temperature and bias voltage. As can be seen, the evolutions of resistance and capacitance as a function of temperature and bias voltage are well accounted for. In particular, the model describes rather well the increase of resistance and the correlated decrease of capacitance as a function of temperature. Also, the opposite variations of $\Delta R / R$ and $\Delta C / C$ with bias voltage are rather well described including their non-linear behavior.

The value obtained for the charging energy $E_{c}=20 \mathrm{meV}$ is in good agreement with the one determined previously [18] for similar ligand molecules. It is comparable to the thermal activation energy (25 $\mathrm{meV}$ ), at room-temperature, which indicates an intermediate Coulomb blockade regime. The fitted value of the total capacitance $C_{\infty}=10 \mathrm{fF}$ is one order of magnitude smaller than the $100 \mathrm{fF}$ obtained (at $10 \mathrm{MHz}$ ) with the same deposition technique, ligand molecules and gold NP diameter [9]. Such a difference can be ascribed to parasitic capacitances not completely eliminated during the calibration phase.

\section{2 - Zero strain plasmonic photo-capacitance:}

Electrical measurements under optical irradiation were performed in order to investigate the coupling between the surface plasmons of the metal nanoparticles and their charge transport properties. 


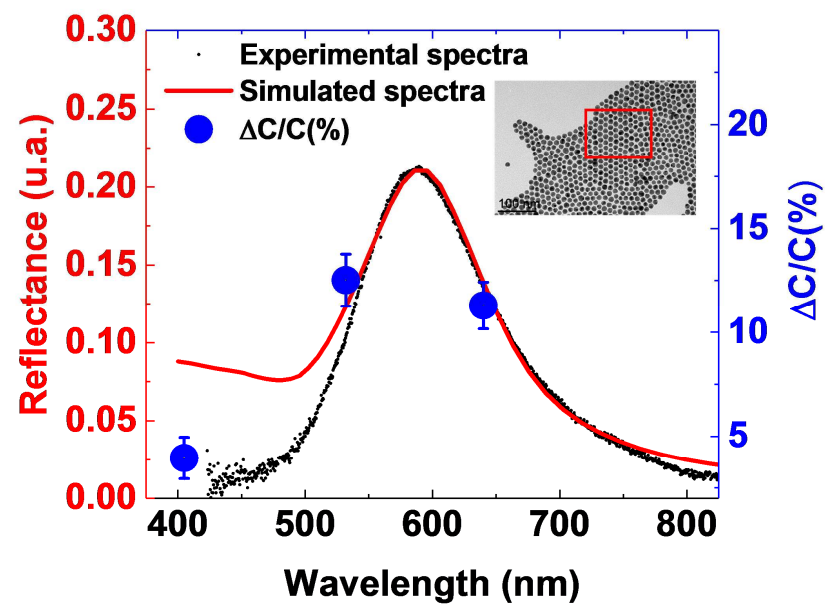

(a)

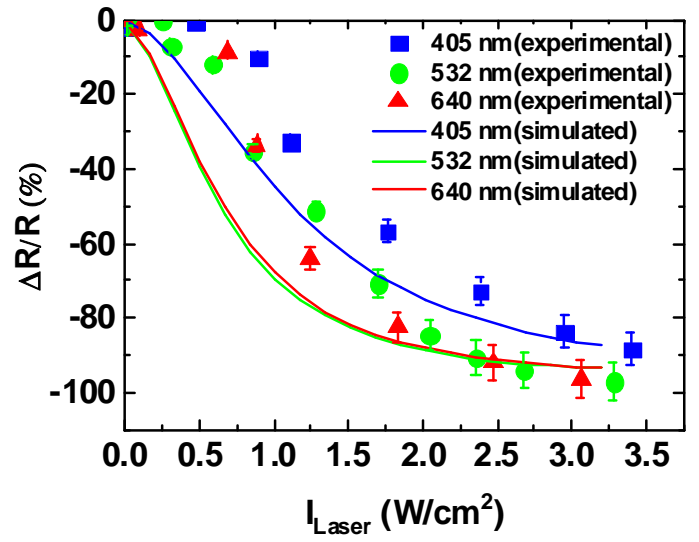

(b)

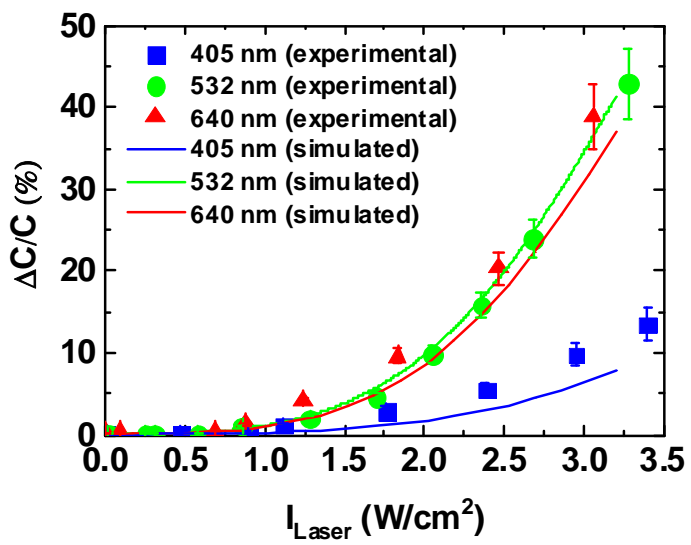

(c)

Figure 3 : (a) Optical reflectance spectrum of the NP assembly (black) compared to the DDA simulated spectrum (red) and to the relative variation $\Delta C / C$ of the capacitance (blue dots) measured as a function of excitation wavelength at $2.2 \mathrm{~W} / \mathrm{cm}^{2}$ laser intensity. Measured (dots) and calculated (lines) variations of the relative resistance (b) and capacitance (c) of the NP assemblies with laser intensity $I_{\text {Laser }}$ increasing from 0 to $3.2 \mathrm{~W} / \mathrm{cm}^{2}$ and for excitation wavelengths $\lambda_{\text {inc }}=405$, $532,640 \mathrm{~nm}$.

We thus focus on the plasmo-electronic response of the NP monolayer, particularly on the capacitance variation induced by the optical excitation.

Figure 3a presents the measured optical extinction spectrum of the NP assembly of Figure 1. A clear plasmonic resonance centered around $600 \mathrm{~nm}$ is observed. Calculations based on the Discrete Dipole Approximation (DDA) were performed in order to ascertain the origin of the optical resonance and to point out the role of the interaction between the metal nanoparticles. To do so, we have performed DDA simulations assuming nearly 120 nanoparticles with the actual positions, sizes and 
inter-particle distances as extracted from the transmission electron microscopy (TEM) images (insert in Figure 3a and Figure ESI 5a). We found a good agreement between the measured and simulated DDA spectra, hence corroborating the plasmonic origin of the observed resonance. It is worthwhile to mention that the interaction between the NPs plays an important role as the plasmonic peak wavelength depends on the inter-particle distance. As a matter of fact, we found that inter-particle distances slightly larger (+10\%) than those measured by TEM, account rather well for the plasmonic peak wavelength and line width (see Figure ESI 5b). This can be due to the variation of the steric interaction between the ligand molecules under ambient conditions compared to the high vacuum TEM environment.

As a matter of fact, according to the plasmonic absorption cross section calculated using the DDA simulations (Figure 3a) and to the nanoparticle density within the self-assembled layer, we estimate that the optical power absorbed by the nanoparticles within the $1 \mathrm{~mm}^{2}$ active array to be around $70 \mathrm{~mW}$. Moreover, assuming that the NP array consists mainly in gold and neglecting the contribution of the surrounding molecules to the thermal conductivity, we estimate the temperature increase due to the photo-thermal heating to be around $2 \mathrm{~K}$. The latter value indicate that, here, the contribution of the photothermal effect to the photoconductance is very weak change compared to previously reported studies where much larger laser intensities (few tens of $\mathrm{kW} / \mathrm{cm}^{2}$ ) were used.

Figure $3 \mathrm{~b}$ and $\mathrm{c}$ display the variations of the relative resistance $\Delta R / R$ and capacitance $\Delta C / C$ as a function of laser intensity and for excitation wavelengths close to the plasmonic resonance. $\Delta R / R$ decreases with increasing laser intensity for all excitation wavelengths. This photo-induced effect has already been reported in previous works [14] and was interpreted as due to the photo-thermal enhanced charge tunneling between nanoparticles, combined with charge trapping of the photogenerated carriers which leads to the non-linear variations observed in Figure 3b. On the contrary, $\Delta C / C$ increases with increasing laser intensity. Although smaller than $\Delta R / R, \Delta C / C$ reaches $43 \%$ at $I_{\text {laser }}$ $=3.2 \mathrm{~W} / \mathrm{cm}^{2}$. As for the temperature and bias voltage (Figure 2), the evolutions of the resistance and the capacitance as a function of laser intensity are opposite. Moreover, these variations depend on the excitation wavelength (Figure $3 \mathrm{~b}, \mathrm{c}$ ). The $\Delta C / C$ measured with the 405,532 and $640 \mathrm{~nm}$ excitation wavelengths (at $2.2 \mathrm{~W} / \mathrm{cm}^{2}$ ) laser intensity are plotted in Figure 3a. A clear correlation between $\Delta C / C$ and the plasmonic resonance of the NP assembly is observed. Indeed, the maximum of $\triangle C / C$ is obtained for $\lambda_{\text {inc }}=532 \mathrm{~nm}$, nearly matching the plasmonic resonance which confirm the plasmonic enhancement of the capacitance Figure 3c. To the best of our knowledge, such effect is reported here for the first time.

The plasmonic effect, on both the resistance and the capacitance of the NP assembly, can be analyzed using the macroscopic circuit model (Figure 1d) in which the change of the conductance due 
to the optical excitation is included through the photo-conductance $G_{\text {photo }}$ term in Equ. (7). As for the effects of temperature and bias voltage, the photo-induced capacitance is deduced from the photoinduced resistance of the NP assembly using Equ. (8). One issue in estimating $G_{\text {photo }}$ is to determine the wavelength dependence of the $k_{\text {photo }}$ term in Equ. (7). To overcome this difficulty, we first assume that $k_{\text {photo }}$ is proportional to the wavelength-dependent optical absorption of the NP assembly and we use the absorption coefficient measured at $405 \mathrm{~nm}$ wavelength as a reference. We thus obtain $k_{\text {photo }}(532 \mathrm{~nm})=3.1 \times k_{\text {photo }}(405 \mathrm{~nm})$ and $k_{\text {photo }}(532 \mathrm{~nm})=2.8 \times k_{\text {photo }}(405 \mathrm{~nm})$. In order to determine $k_{\text {photo }}(405 \mathrm{~nm})$, we compare the measured and calculated variations of $\Delta R / R$ and $\Delta C / C$ as a function of laser intensity at $405 \mathrm{~nm}$ excitation wavelength and use $k_{\text {photo }}(405 \mathrm{~nm})$ as the only fitting parameter; all other parameters $\left(N_{j}, E_{C}\right.$ and $\left.C_{\infty}\right)$ are fixed to their previously determined values (Figure 2$)$. This fitting procedure is performed for $\Delta R / R$ and $\Delta C / C$, simultaneously and the correlation between resistance and capacitance is still given by Equ. (8). We thus obtain $k_{\text {photo }}(405 \mathrm{~nm}) \sim 5 \mathrm{pS} . \mathrm{cm}^{2} / \mathrm{kW}$. Close to the plasmonic resonance (i.e. $532 \mathrm{~nm}$ ) $\mathrm{k}_{\text {photo } \mid 532 \mathrm{~nm}} \sim 16 \mathrm{pS} . \mathrm{cm}^{2} / \mathrm{kW}$. The latter value is smaller than the one $35.5 \mathrm{pS} . \mathrm{cm}^{2} / \mathrm{kW}$ reported in Gauvin et al. [14]. Such a difference may arise from, the fact that the NP assembly is here supported by the polyimide substrate and not suspended as in [14] which may impact the overall absorption of the nanoparticle array and hence the $\mathrm{k}_{\text {photo }}$ parameter. Figure $3 \mathrm{~b}$ and Figure $3 \mathrm{c}$ show that the calculations account rather well for the experimental variations of both $\Delta \mathrm{R} / \mathrm{R}$ and $\Delta \mathrm{C} / \mathrm{C}$ with the laser intensity. The deviations, between calculated and measured $\Delta \mathrm{R} / \mathrm{R}$ and $\Delta \mathrm{C} / \mathrm{C}$ at low laser irradiation, arise from the linear approximation of $\mathrm{G}_{\text {photo }}$ used in Eq.7. It is worth to underline that the photo-induced electric response of the NP assembly is here introduced through the parameter $G_{p h o t o}$ (Equ. (7)) which contains the physics of the plasmonic-to-electric energy conversion. This allows to account for the effect of the plasmonic pumping of electrons and holes on the inter-particle admittance (Equ. (1)) and finally on the overall resistance $R$ of the nanoparticle assembly (Equ.(6)).

\subsection{Strain induced plasmonic photo-capacitance:}

The NP assembly is formed on a stretchable substrate offering a unique mean of monitoring the inter-particle distances and hence their electronic and plasmo-electronic properties. Figure 4a shows capacitance measurements performed under laser illumination at $0.85 \mathrm{~W} / \mathrm{cm}^{2}$, for 405,532 and $640 \mathrm{~nm}$ excitation wavelengths, as a function of applied extensive strain ranging from 0 to $1.5 \%$ (elastic domain of polyimide). As can be noticed, the variation of $\Delta C / C$ with strain is linear, whatever the optical conditions. Under dark conditions (no illumination), the measured gauge factor $G F_{C}=\frac{\Delta C / C}{\varepsilon}$ is around -1.2. Remarkably, it is enhanced by nearly a factor 3 under laser illumination and for the excitation wavelengths nearly matching the plasmonic resonance (i.e. 532 and $640 \mathrm{~nm}$ ). For the nonmatching wavelength $(405 \mathrm{~nm})$, the enhancement is only a factor 2 . 
Measurements of the gauge factor in dark conditions were performed before and after illumination at $0.85 \mathrm{~W} / \mathrm{cm}^{2}$ following the sequence: dark, red laser, dark, green laser, dark, blue laser (Figure 4a). All the curves recorded under dark conditions systematically overlap, which indicates that there is no degradation of the NP assembly upon laser irradiation or mechanical deformation. Irradiations with much larger laser intensities produce the melting and the coalescence of the nanoparticles [14].

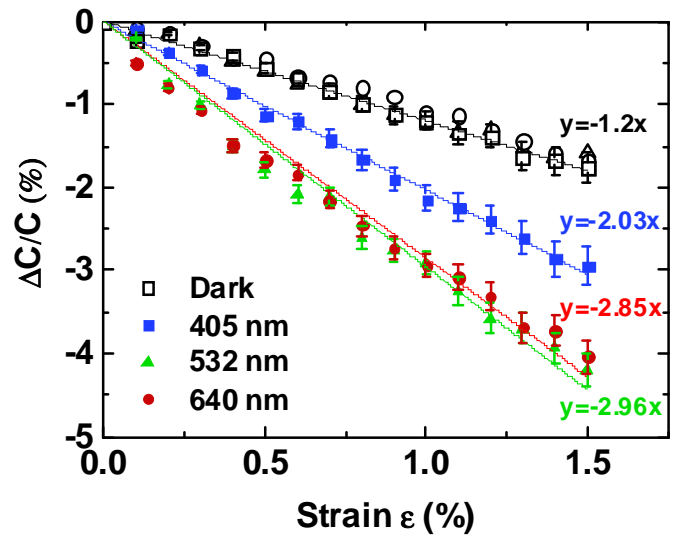

(a)

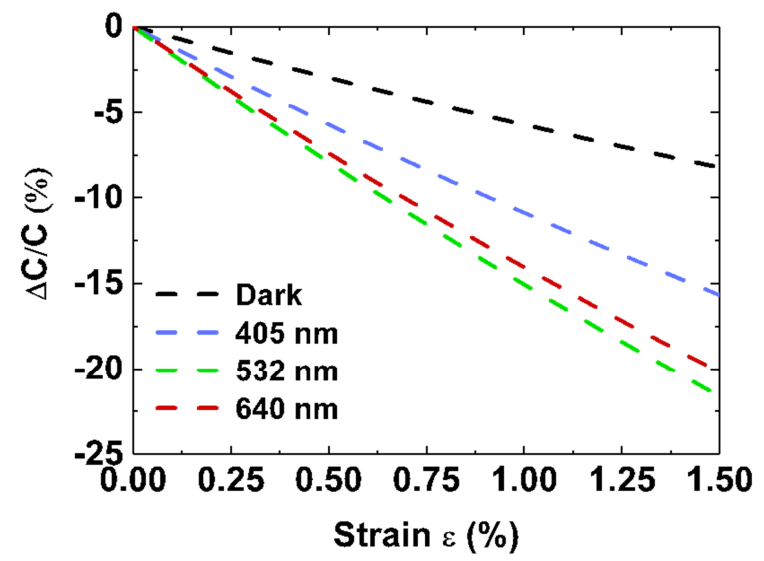

(b)

Figure 4: Measured (a) and simulated (b) Relative capacitance $\triangle C / C$ of the NP assembly as a function of applied strain under dark conditions and for the excitation wavelengths $\lambda_{\text {inc }}=405,532$, $640 \mathrm{~nm}$. The laser intensity is set at $0.85 \mathrm{~W} / \mathrm{cm}^{2}$. The lines in (a) show the results of a least-square linear fit which allow to extract the experimental gauge factors: $G_{f_{c}}($ dark $)=-1.2, G_{f c}(405 \mathrm{~nm})=-$ $2.03, G_{f c}(532 \mathrm{~nm})=-2.96$ and $G_{f c}(640 \mathrm{~nm})=-2.85$.

To gain a fundamental understanding of the plasmo-electro-mechanical properties of the NP assembly, we have performed numerical simulations based on the model introduced in section 2. As discussed previously, the inter-particle local resistance $R_{i j}$ (Equ. (2)) and capacitance $C_{i j}$ (Equ. (4)) both depend on strain through the inter-particle spacing. The uniaxial ( $x$ axis) applied strain $\varepsilon$ is introduced in the numerical simulations by shifting the NP coordinates according to:

$$
\text { (9) } \mathrm{x}_{\mathrm{i}}, \mathrm{y}_{\mathrm{i}} \rightarrow \mathrm{x}_{\mathrm{i} \cdot}(1+s . \varepsilon), \mathrm{y}_{\mathrm{i} \cdot} \cdot\left(1-v_{\text {poisson }} . \mathrm{s. \varepsilon}\right)
$$

where $v_{\text {poisson }}=0.37$ is the Poisson's coefficient of the polyimide substrate, and $s$ is the substrate-tolayer strain transmission factor (stick/slip) which is, in a first step, assumed to be 1 since the NP assembly is here formed by a single monolayer.

The inter-particle resistance $R_{i j}$ and capacitance $C_{i j}$ are calculated according to the strained NP network. The total resistance $R$ and capacitance $C$ are then computed using the numerical 
resolution of Equ. (1)-(5) as discussed above. The simulations were performed for strain ranging from 0 to $1.5 \%$ with and without optical excitation at $0.85 \mathrm{~W} / \mathrm{cm}^{2}$. The results are presented in Figure $4 \mathrm{~b}$.

Under dark conditions, the relative capacitance $\Delta C / C$ decreases linearly with increasing strain as observed experimentally (Figure 4a) and in agreement with Equ.(4) which indicates that the interparticle capacitance decreases linearly with increasing inter-particle distance [9]. Under illumination, the gauge factor increases due to the photo-induced inter-particle conductance ( $G_{\text {photo }}$ term in Equ. (7)) which impacts the overall resistance and thus the overall capacitance (Equ. (8)) of the NP assembly. Using the values of $k_{\text {photo }}$ determined previously (section 3.3), the calculated $G F_{c}$ is increased by a factor 3 (compared to the dark conditions) for the 532 and $640 \mathrm{~nm}$ wavelengths, i.e., close to the plasmonic resonance, and by a factor 2 for the $405 \mathrm{~nm}$ wavelength, in agreement with the measurements (Figure 4a). However, the calculations overestimate the absolute values of $\Delta C / C$ by a nearly a factor 5 . This can be explained by three main reasons. First, the strain within the NP assembly could be overestimated. Indeed, in Equ.(9) the substrate-to-layer transmission coefficient is assumed to be 1 , which could not be the case due to the weak adhesion of the layer on the substrate, as already observed for resistive strain gauges [32]. Second, a rearrangement of the ligand molecules within the strained NP network may change the dielectric constant surrounding the metal NPs and hence the inter-particle capacitance (Equ. (4)). In order to investigate these two effects, we have performed numerical simulations of $\Delta C / C$ for various strain transmission coefficients ( $s=1$ to 0.7 , Equ.(9)) and dielectric constants (ranging from 2.3 to 2.32, Equ. (4)). As expected, both effects are responsible for a decrease of the sensitivity of $\Delta C / C$ to the applied strain (see Figure ESI 7). Finally, the applied stress may also be partially accommodated by disorder effects due to size and inter-particle distance fluctuations, as well as to defects in the NP arrangement within the network, thus limiting the strain of the NP assembly.

We have shown that the change of resistance $\mathrm{R}$, in turn, translates into a photo-capacitance through Equ.(8). However, as mentioned above, the impact of the optical excitation on the local photoconductance gphoto is not introduced explicitly in equation 1. To do so, a description, at the microscopic level, of the conversion of surface plasmons into charge carriers and current in the selfassembled nanoparticle array is required. For instance, the effect of optical excitation of the interparticle capacitance could be introduced as an AC plasmonic voltage superimposed on the applied DC voltage [31]. The local electric field and potential may be enhanced by the plasmonic resonance and by the near-field interaction between the nanoparticles. In that case, one may expect a direct plasmonic contribution to the local capacitance and hence to the overall photocapacitance of the nanoparticle array. Also, the interparticle resistance may include a contribution which accounts for the decay of the surface plasmons into electrons (and holes) and their transport through tunneling effect and charge transfer via electron state hybridization between the metal nanoparticle and the coating molecules. 
Moreover, under laser irradiation, the ligand molecules pinned to the nanoparticle surface, undergo a quite complex adsorption/desorption dynamics [33] which may impact the charge tunneling and transfer and hence the overall photo-resistance and photo-capacitance.

\section{Conclusions:}

In summary, we have investigated the dependence on temperature, bias voltage, optical excitation and strain of the electronic transport properties of self-assembled gold nanoparticle monolayers. A special attention has been paid to the correlation between resistance and capacitance as both characteristic bring complementary information on the charge transport phenomenon in these complex molecules/nanoparticle hybrid systems. The interpretation of the experimental results was achieved using a detailed modelling of the charge transport both at the nanoscale and at the macroscopic scale of the NP assembly. The charge tunnelling and hopping between nanoparticles were describded using a nano-circuit junction with temperature, bias voltage and strain dependent resistance and capacitance. The effect of the optical excitation and transduction of the surface plasmons into an electric current was introduced as an additional photo-induced term to the macroscopic impedance. To connect the nanoscale and the macroscopic scale, the overall resistance and capacitance of the NP assembly were determined using numerical simulations. Our main findings can be summarized as follows: (i) the correlation between resistance and conductance characteristics was pointed out experimentally and interpreted using a quantitative analysis of the measurements based on the relation between the macroscopic resistance and capacitance. In particular, we found that the relative change of the capacitance can be as large as $43 \%$ at the surface plasmon resonance, which paves the way to applications of self-assembled gold NP monolayers as highly sensitive photo-capacitance sensors. (ii) the modification of the charge transport through the NP assembly under optical excitation and simultaneous applied stress showed that the capacitance strain gauge factor is increased by a factor 3 at the surface plasmon resonance, in agreement with the calculations. This result may be exploited for optically enhanced capacitance strain-sensors. The conversion of surface plasmons into electron-hole pairs transported throughout a network of nanoparticles is a complex multi-scale phenomenon. It requires a deep fundamental understanding of the interaction mechanisms underlying the generation and decay of the involved excitations, but also a detailed description of the molecule/nanoparticle charge transfer (hybridization, tunnelling, hopping and trapping). Our work contributes to bridge the gap between fundamental investigations of the optoelectronic properties of NP assemblies and their practical use as highly sensitive photo-resistance and photo-capacitance sensors.

\section{Acknowledgements}


This work was supported by "Initiatives d'excellence IDEX UNITI" in the framework of its program “Actions Thématiques Stratégiques" AAP 2015 (Project "WE CAN"). This work received computational support from HPC center CALMIP at Toulouse university.

\section{Data availabitity:}

The raw/processed data required to reproduce these findings cannot be shared at this time due to technical or time limitations. The raw data required to reproduce these findings will be available to download very quickly from [http://lpcno.insa-toulouse.fr/spip.php?article1588\&lang=en]. The processed data required to reproduce these findings will be available to download very quickly from [http://lpcno.insa-toulouse.fr/spip.php?article1588\&lang=en].

\section{References:}

[1] H. Nakanishi et al. Nature, 2009, 460, 371-375.

[2] M. A. Mangold et al. Appl. Phys. Lett., 2009, 94, 161104.

[3] M. A. Mangold et al. J. Am. Chem. Soc., 2011, 133, 12185-12191.

[4] Ambrosetti, A., Ferri, N., DiStasio, R.A., and Tkatchenko, A. (2016). Wavelike charge density fluctuations and van der Waals interactions at the nanoscale. Science 351, 1171-1176.

[5] Y. Zhang, O. Dai, M. Levy, and M. P. Sarachik, "Probind the Coulomb Gap in Insulating n-Type CdSe," Phys. Rev. Lett., vol. 64, no. 22, p. 2687, 1990.

[6] J. Herrmann et al., "Nanoparticle films as sensitive strain gauges," Appl. Phys. Lett., vol. 91, no. 18, p. $183105,2007$.

[7] J. Grisolia, N. Decorde, M. Gauvin, N. M. Sangeetha, B. Viallet, and L. Ressier, 'Electron transport within transparent assemblies of tin-doped indium oxide colloidal nanocrystals," Nanotechnology, vol. 26, no. 33, p. 335702, 2015.

[8] M. M. A. Yajadda,1,2 K.-H. Müller,2 and K. Ostrikov2,1 "Effect of Coulomb blockade, gold resistance, and thermal expansion on the electrical resistance of ultrathin gold films"PHYSICAL REVIEW B 84, 235431 (2011)

[9] H. Nesser, J. Grisolia, T. Alnasser, B. Viallet and L. Ressier: "Towards wireless highly sensitive capacitive strain sensors based on gold colloidal nanoparticles" Nanoscale, 2018,10, 10479-10487

[10] D. S. Bychanok, A. G. Paddubskaya, P. P. Kuzhir, S. A. Maksimenko, C. Brosseau, J. Macutkevic, and S. Bellucci : "A study of random resistor-capacitor-diode networks to assess the electromagnetic properties of carbon nanotube filled polymers" Appl. Phys. Lett. 103, 243104 (2013); https://doi.org/10.1063/1.4847335

[11] H. Hiramatsu and F. E. Osterloh, "A simple large-scale synthesis of nearly monodisperse gold and silver nanoparticles with adjustable sizes and with exchangeable surfactants," Chem. Mater., vol. 16, no. 13, pp. 2509-2511, 2004.

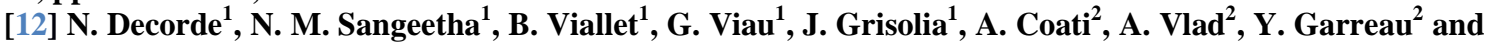
L. Ressier" "Small Angle X-ray Scattering Coupled With in-situ Electromechanical Probing of Nanoparticle-Based Resistive Strain Gauges" Nanoscale, (2014), 6, 15107-15116 DOI: 10.1039/C4NR04129A.

[13] A. J. Quinn et al., "Manipulating the charging energy of nanocrystal arrays," Small, vol. 1, no. 6, pp. 613-618, 2005.

[14] M. Gauvin et al., "Plasmonic photo-current in freestanding monolayered gold nanoparticle membranes," Nanoscale, vol. 8, no. 36, pp. 16162-16167, 2016.

[15] M. A. Mangold, M. Calame, M. Mayor and A. W. Holleitner, J. Am. Chem. Soc., 2011, 133, 1218512191.

[16] Scilab Enterprises (2012). Scilab: Free and Open Source software for numerical computation (OS, Version 5.XX) [Software]. Available from: http://www.scilab.org 
[17] P. Lagonotte et Y. Eichenlaub, « Réseaux électrocinétiques et algèbre linéaire (notions fondamentales) », J3eA, vol. 5, mai 2006.

[18] H. Moreira, J. Grisolia, N. M. Sangeetha, N. Decorde, C. Farcau, B. Viallet, K. Chen, G. Viau and L. Ressier, "Electron transport in gold collö̈dal nanoparticle based strain gauges", Nanotechnology 24 (2013) 095701 (9pp).

[19] Yajadda, M. M. A.; Müller, K.-H.; Ostrikov, K. 2011 Phys. Rev. B 84235431.

[20] http://www.goodfellow.com/A/Polyimide.html

[21] Zabet-Khosousi, A.; Dhirani, A.-A. 2008 Chem. Rev. 1084072.

[22] K. -H. Müller, G. Wei, B. Raguse, and J. Myers Phys. Rev. B 68, 155407 (2003).

[23] Müller, K.-H.; Herrmann, J.; Raguse, B.; Baxter, G.; Reda, T. 2002 Phys. Rev. B 66075417.

[24] K.-H. Müller and M. M. A. Yajadda Journal of Applied Physics 111, 123705 (2012); doi: 10.1063/1.4729491.

[25] H. Moreira, Q. Yu, B. Nadal, B. Bresson, M. Rosticher, N. Lequeux, A. Zimmers, and H. Aubin, Phys. Rev. Lett. 107, 176803 (2011).

[26] Müller K-H, Herrmann J, Wei G, Raguse B and Wieczorek L 2010 ICONN: Int. Conf. on Nanoscience and Nanotechnology page(s) 316-318, 22-26 Feb. 2010, DOI : 10.1109/ICONN.2010.6045236, Publication Year: 2010.

[27] A. Goswami, Amit P. Goswami, Dielectric and optical properties of ZnS films, Thin Solid Films 16 (1973) 175-185

[28] G.B. Sakr, I.S. Yahia: "Effect of illumination and frequency on the capacitance spectroscopy and the relaxation process of $\mathrm{p}-\mathrm{ZnTe} / \mathrm{n}-\mathrm{CdMnTe} / \mathrm{GaAs}$ magnetic diode for photocapacitance applications" Journal of Alloys and Compounds 503 (2010) 213-219.

[29] I.S.Yahia, F.Yakuphanoglu, Osama A.Azim : "Unusual photocapacitance properties of a monocrystalline silicon solar cell for optoelectronic applications" Solar Energy Materials \& Solar Cells 95 (2011) 2598-2605

[30] Link, S.; El-Sayed, M. A. Spectral properties and relaxation dynamics of surface plasmon electronic oscillations in gold and silver nanodots and nanorods. J. Phys. Chem. B. 1999, 103, 8410-8426.

[31] M. M. A. Yajadda, K.-H. Müller, D. I. Farrant, and K. Ostrikov, Partial rectification of the plasmoninduced electrical tunnel current in discontinuous thin gold film at optical frequency. Appl. Phys. Lett. 100, 211105 (2012); https://doi.org/10.1063/1.4720513

[32] N. Decorde1, N. M. Sangeetha1, B. Viallet1, G. Viau1, J. Grisolia1, A. Coati2, A. Vlad2, Y. Garreau2 and L. Ressier1. "Small Angle X-ray Scattering Coupled With in-situ Electromechanical Probing of Nanoparticle-Based Resistive Strain Gauges" Nanoscale, (2014), 6, 15107-15116 DOI: 10.1039/C4NR04129A.

[33] Amine Mezni, Thameur Dammak, Anis Fkiri, Adnen Mlayah, Younes Abid, and Leila Samia Smiri. Photochemistry at the Surface of Gold Nanoprisms from Surface-Enhanced Raman Scattering Blinking, J. Phys. Chem. C 118, 31, 17956-17967 


\title{
Electronic Supplementary Information
}

\section{Plasmonic photo-capacitance of self-assembled gold colloidal nanoparticle monolayers}

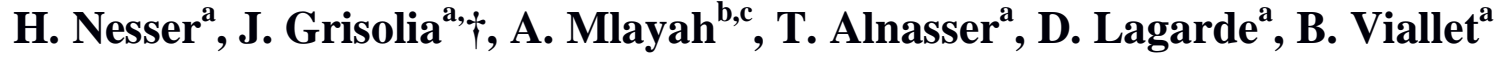 \\ and L. Ressier ${ }^{\mathrm{a}}$ \\ a. LPCNO, Université de Toulouse, INSA, CNRS, UPS, 135 avenue de Rangueil, Toulouse 31077, France. \\ b. CEMES, Université de Toulouse, CNRS, UPS, Toulouse, France \\ c. LAAS, Université de Toulouse, CNRS, UPS, Toulouse, France \\ $\dagger$ Corresponding author: jeremie.grisolia@insa-toulouse.fr
}

\section{Sample fabrication process}

Interdigitated gold electrodes were fabricated on $125 \mu$ m polyimide (Upilex) films (Figure ESI 1a).using the lift-off method (Figure ESI 1c). A reverse pattern was created in the sacrificial stencil layer by laser photolithography. Then, a $50 \mathrm{~nm}$ gold Au layer was deposited over the entire surface of the substrate by IBS-E ion beam sputtering (Figure ESI 1d). After lift-off, $1 \mathrm{~mm}^{2}$ sensors with 50 pairs of interdigitated electrodes $5 \mu \mathrm{m}$ wide and $5 \mu \mathrm{m}$ interdigitated distance was obtained and connected by external gold electrodes (Figure ESI 1e).

$14 \mathrm{~nm}$ gold nanoparticles functionalized with dodecanethiol (C12) organic ligands were deposited between on the interdigitated electrodes by the convective assembly technique. A $19 \mu \mathrm{L}$ of a colloidal suspension of gold NPs in toluene was dropped on top of a $60 \mu \mathrm{L}$ ethylene glycol (EG) droplet located at the surface lever of the substrates (Figure ESI 1f). EG was preferred because of its high viscosity and low evaporation rate which improve the long range ordering of the gold NPs. The gold NPs selfassemble as a close-packed ordered array at the surface of the EG droplet (Figure ESI 1f). As the EG droplet slowly evaporated, over $24 \mathrm{~h}$ under mild heating at $55^{\circ} \mathrm{C}$, the gold NP array was transferred onto the surface of the substrate, hence covering the entire active zone. 


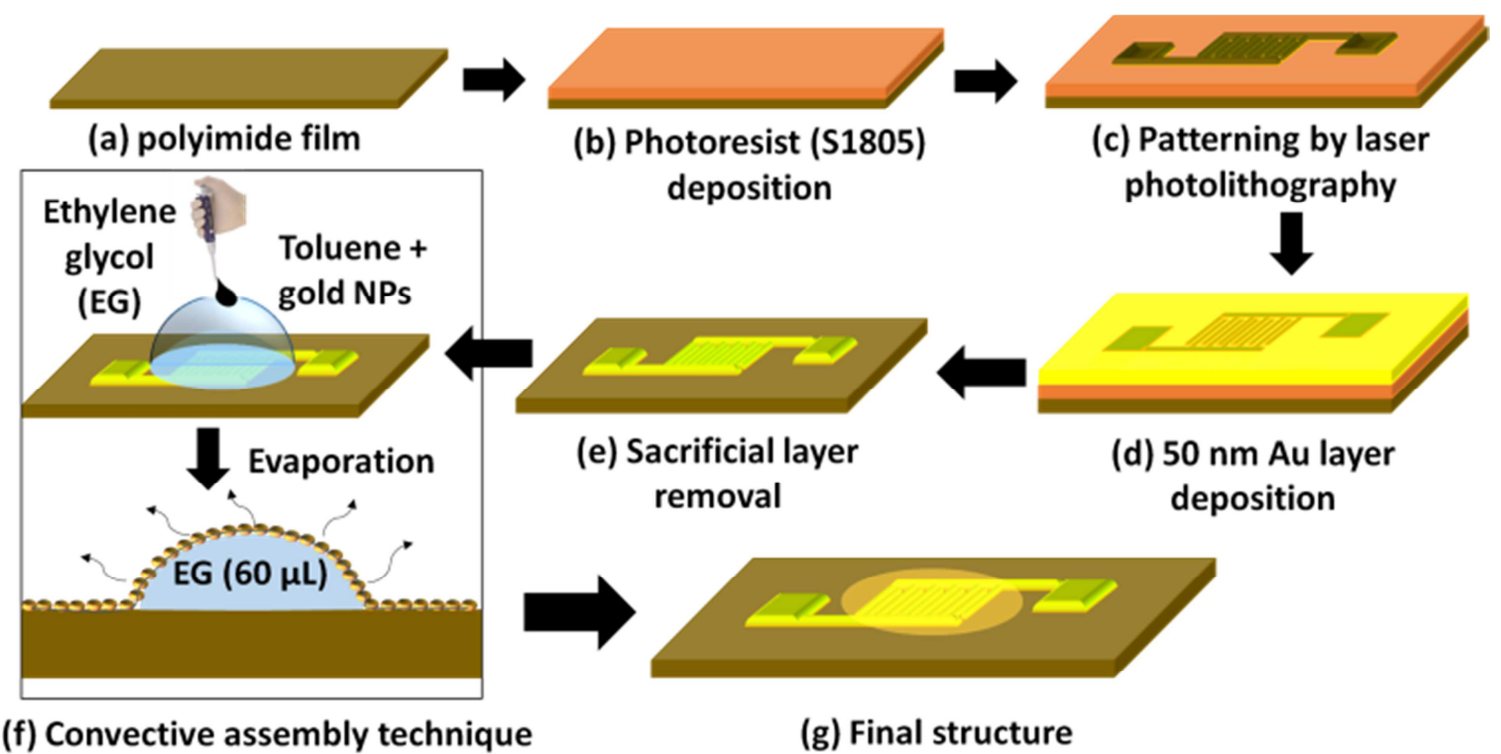

Figure ESI 1 : Fabrication process of the NP self-assembled samples

\section{II - Equivalent electrical circuit}

The overall transport properties of the NP assemblies can be derived by from an equivalent circuit made of a parallel R-C resistance and capacitance, and a bulk resistance $r_{s}$ in series due in particular to lead lengths. However, we can consider that the electrode resistance $r_{s}$ is negligible with respect to the total resistance of the NP assembly (few $\Omega$ compared to few M $\Omega$ ).

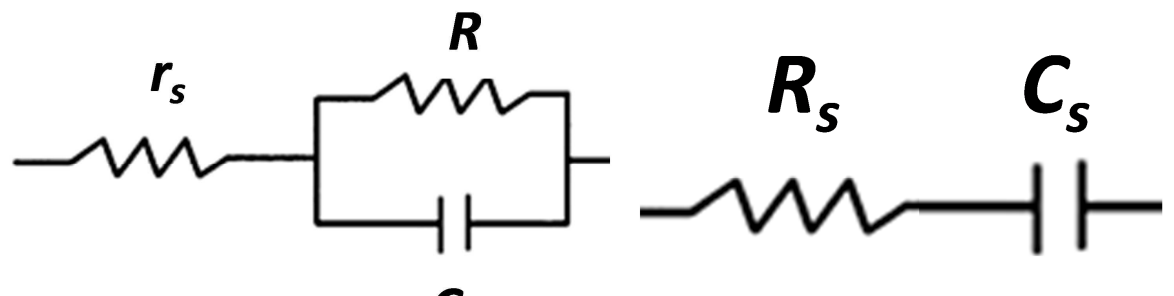

(a)

(b)

Figure ESI 2 : (a) Equivalent electrical circuit elements ( $R, C$, and $r_{s}$ ) (b) Equivalent series circuit $\left(\mathbf{C}_{\mathrm{S}}\right.$ and $\left.\mathbf{R}_{\mathrm{S}}\right)$.

The impedance $\mathrm{Z}$ of the circuit (Figure ESI 2a) can be written:

(1) $Z=r_{S}+\frac{R}{1+j \omega R C}=\frac{R+r_{S} \cdot\left(1+\omega^{2} R^{2} C^{2}\right)}{1+\omega^{2} R^{2} C^{2}}-\frac{j \omega R^{2} C}{1+\omega^{2} R^{2} C^{2}}$ 
In order to extract the resistive and capacitive macroscopic characteristics of the circuit, it is useful to look for the equivalent series circuit. This impedance can also be represented in a simpler equivalent series circuit (Figure ESI 2b):

$$
\text { (2) } Z=R_{s}-j \frac{1}{\omega C_{s}}
$$

By matching the real and imaginary parts of the two previous equations, we find the relation between resistance $R$ and capacitance $C$ and $R s$ series resistance and $C s$ serie capacitance:

$$
\text { (3) } R_{S}=\frac{R+r_{S} \cdot\left(1+\omega^{2} R^{2} C^{2}\right)}{1+\omega^{2} R^{2} C} \text { and } C_{S}=\frac{1+\omega^{2} R^{2} C^{2}}{\omega^{2} R^{2} C}
$$

It is worth noting that when the condition $1 / \omega R^{2} C<<C$ is satisfied, the series capacitance $\mathrm{C}_{\mathrm{s}}$ tends towards the parallel capacitance $\mathrm{C}$ value, which is fulfilled which is fulfilled in our study, since, we have $\frac{1}{\omega R^{2} C}=8 \times 10^{-13} \mathrm{~F}<<C=3 \times 10^{-12} \mathrm{~F}$ (by roughly taking $\mathrm{f} \sim 1 \times 10^{6} \mathrm{~Hz}, \mathrm{R}=250 \mathrm{M} \square, \mathrm{C} \sim 3 \times 10^{-12} \mathrm{~F}$ ).

\section{III - Capacitance versus Temperature:}

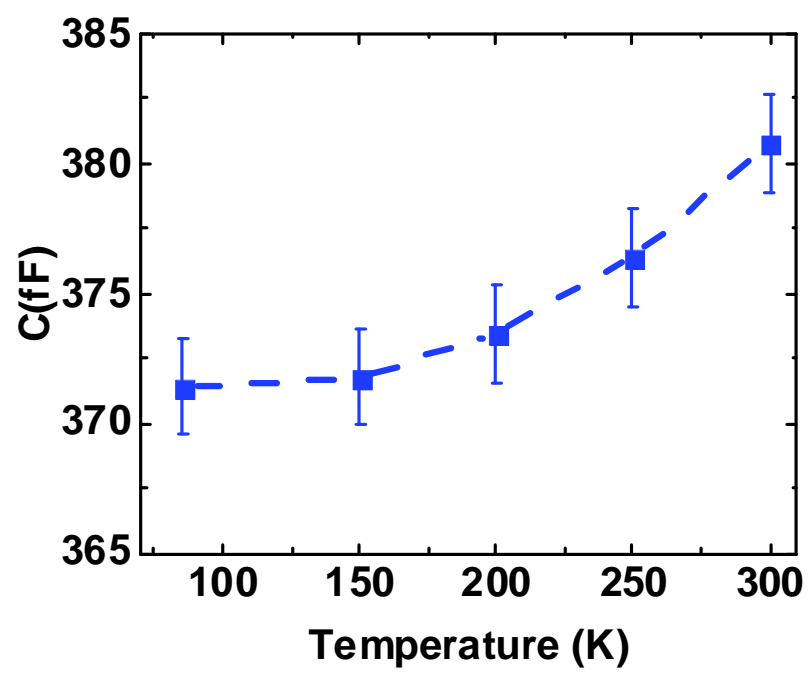

Figure ESI 4 : Capacitance variation versus temperature of the NP self-assembled sample at $\mathbf{f}=500 \mathrm{kHz}$.

\section{V - Discrete dipole approximation (DDA) simulations}

The simulations of the optical extinction spectra and electric near-field were performed using the ddscat 7.3 software by Draine and Flatau [8]-[9]. The positions and sizes of each nanoparticle were extracted from the transmission electron microscope (TEM) image (Figure ESI 5a) assuming spherical nanoparticles. Each nanoparticle was discretized by a cubic mesh of nearly 2000 point dipoles distributed with inter-dipole separation as small as $0.5 \mathrm{~nm}$ in order to ensure full convergence of the 
calculations. The optical index of gold was taken from [5] and the optical index 1.46 of dodecanethiol was assumed for the surrounding medium. As shown in Figure ESI 5b, the plasmonic resonance occurs around $600 \mathrm{~nm}$. In order to estimate the effect of the electro-magnetic near-field interactions, the separation between the nanoparticles was artificially increased while keeping the same sizes and arrangement. In that situation, the surface plasmon resonance is now blue shifted to $540 \mathrm{~nm}$ (Figure $5 b)$ as expected for isolated spherical gold nanoparticle.

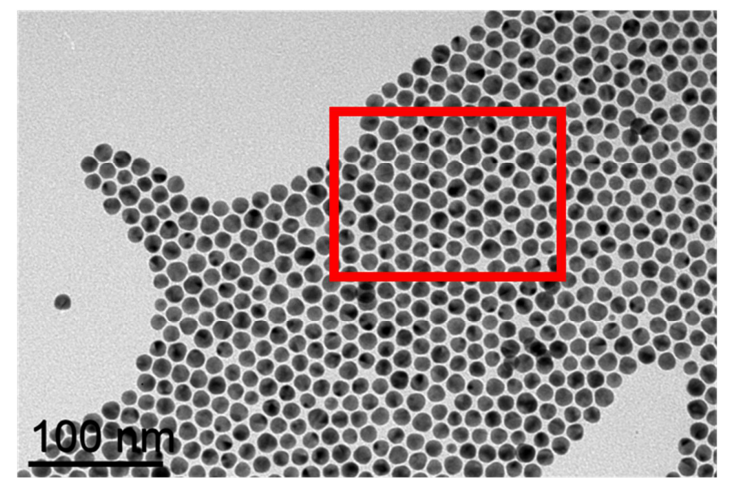

(a)

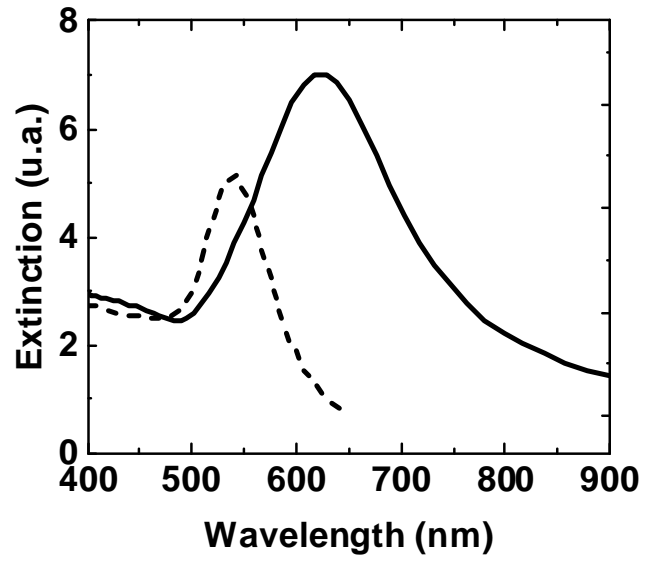

(b)

Figure ESI 5: (a) Transmission electron microscopy image of the NPs assembly (b), full line DDA calculated spectra of the NP assembly located within the square region shown in (a). The dashed line shows the spectrum calculated for the isolated nanoparticle assembly.

\section{VI - Numerical simulations of photo-capacitance}

Numerical simulations of the charge transport through the NP assembly were performed using a home-made Scilab routine. To mimic the actual NPs arrangement, the NP assembly was modeled by a $900 \mathrm{~nm} \times 500 \mathrm{~nm}$ monolayer of regularly packed nanoparticles forming a hexagonal network. All NPs have a diameter of $14 \mathrm{~nm}$, and are separated by a surface-to-surface distance of $1.2 \mathrm{~nm}$ corresponding to $C_{12}$ ligand length at zero strain (Figure ESI 6). 


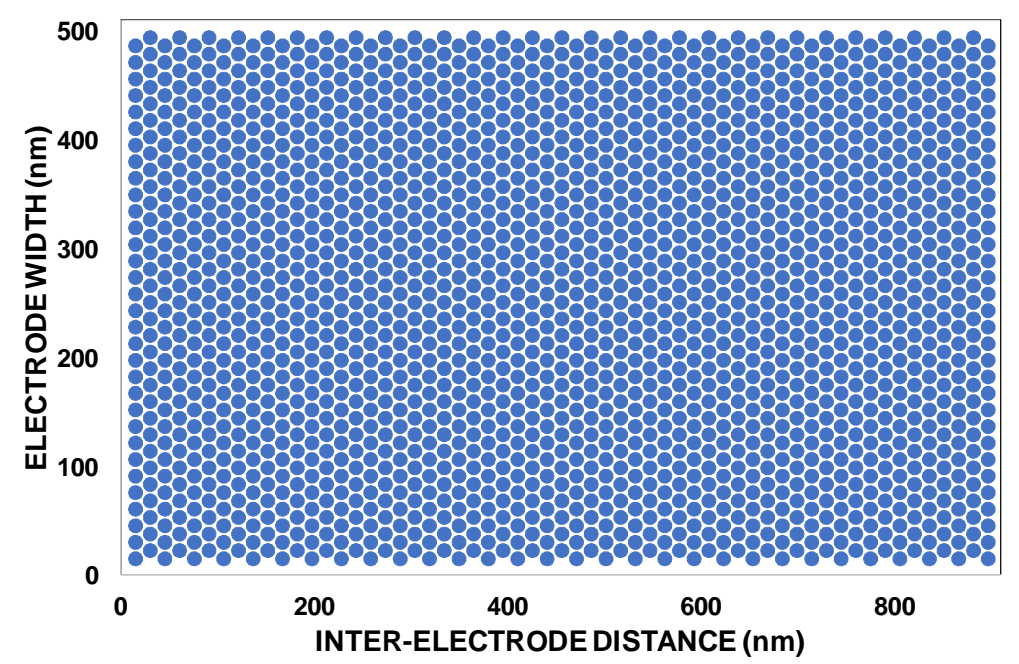

Figure ESI 6 : Hexagonal regular arrangement of the NP assembly used in the numerical simulations.

To introduce the strain, the nanoparticles positions are shifted according to Equ. 8 and the stick/slip $s$ can be varied. The relative permittivity $\varepsilon_{r}$, which appears (Eq. 3) in the capacitance $C_{i j}$ of the inter-particle junction model can also be changed.

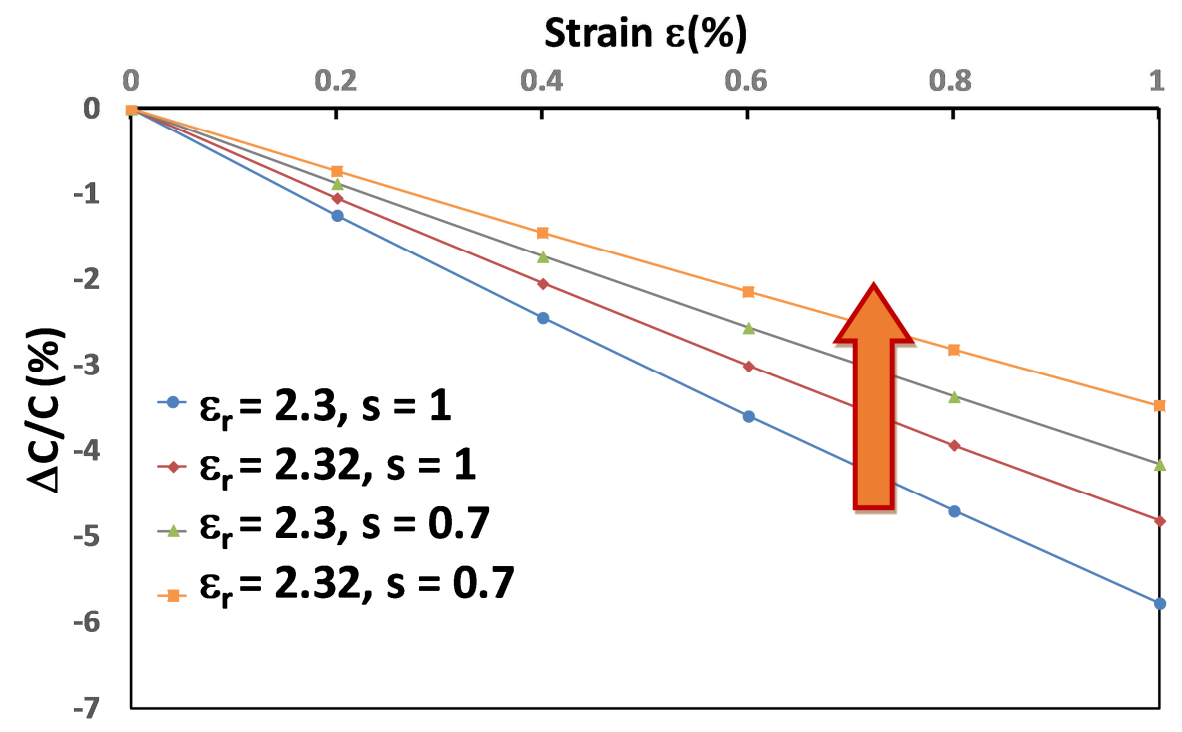

Figure ESI 7 : Simulated variation of the relative capacitance for stick/slip coefficient $s=1$ (full substrateto-layer strain transmission) and $s=\mathbf{0 . 7}$. Simulations were also performed for permittivity $\varepsilon_{r}=\mathbf{2 . 3}$ and 2.32 while keeping the stick/slip coefficient $s=1$.

The relative capacitance is very sensitive to the change in relative permittivity. Indeed, increasing $\varepsilon_{r}$ from 2.3 to 2.32 , which is less than $1 \%$ variation, decreases the gauge factor $G_{f c}$ from -5.8 to $-4.9 \%$. Similarly, reducing the stick/slip coefficient from 1 to 0.7 , decreases the gauge factor from - 
5.8 to $-4.2 \%$. By cumulating the effect of $\varepsilon_{r}$ (from 2.3 to 2.32 ) and $s$ (from 1 to 0.7 ) we decrease even more the gauge factor $G_{f c}$ from -5.8 to $-3.6 .9 \%$.

\section{References ESI:}

[1] T. Vossmeyer, C. Stolte, M. Ijeh, A. Kornowski, and H. Weller, "Networked Gold-Nanoparticle Coatings on Polyethylene: Charge Transport and Strain Sensitivity,” Adv. Funct. Mater., vol. 18, no. 11, pp. 1611-1616, 2008.

[2] M. Gauvin et al., "Electro-mechanical sensing in freestanding monolayered gold nanoparticle membranes," Nanoscale, vol. 8, no. 22, pp. 11363-11370, 2016.

[3] M. Gauvin et al., "Plasmonic photo-current in freestanding monolayered gold nanoparticle membranes," Nanoscale, vol. 8, no. 36, pp. 16162-16167, 2016.

[4] Draine, B. T., 1988. "The Discrete-Dipole Approximation and its Application to Interstellar Graphite Grains". Astrophys. J., 333, 848-872.

[5] Handbook of Optical Constants in Solids Edited by Edward D. Palik (c) Copyright 1985 by Academic press.

[6] A. Goswami, Amit P. Goswami, Dielectric and optical properties of ZnS films, Thin Solid Films 16 (1973) 175-185

[7] G.B. Sakr, I.S. Yahia: "Effect of illumination and frequency on the capacitance spectroscopy and the relaxation process of $\mathrm{p}-\mathrm{ZnTe} / \mathrm{n}-\mathrm{CdMnTe} / \mathrm{GaAs}$ magnetic diode for photocapacitance applications" Journal of Alloys and Compounds 503 (2010) 213-219.

[8] Draine, B. T. \& Flatau, P. J., 1994. "Discrete-dipole approximation for scattering calculations". J. Opt. Soc. Am., 11, 1491-1499.

[9] Flatau, P. J. \& Draine, B. T., 2012. "Fast near-field calculations in the discrete dipole approximation for regular rectilinear grids". Optics Express, 20, 1247-1252.

[10] "Small Angle X-ray Scattering Coupled With in-situ Electromechanical Probing of NanoparticleBased Resistive Strain Gauges” N. Decorde, N. M. Sangeetha, B. Viallet, G. Viau, J. Grisolia, A. Coati, A. Vlad, Y. Garreau and L. Ressier. Nanoscale, (2014), 6, 15107-15116 DOI: 10.1039/C4NR04129A. 


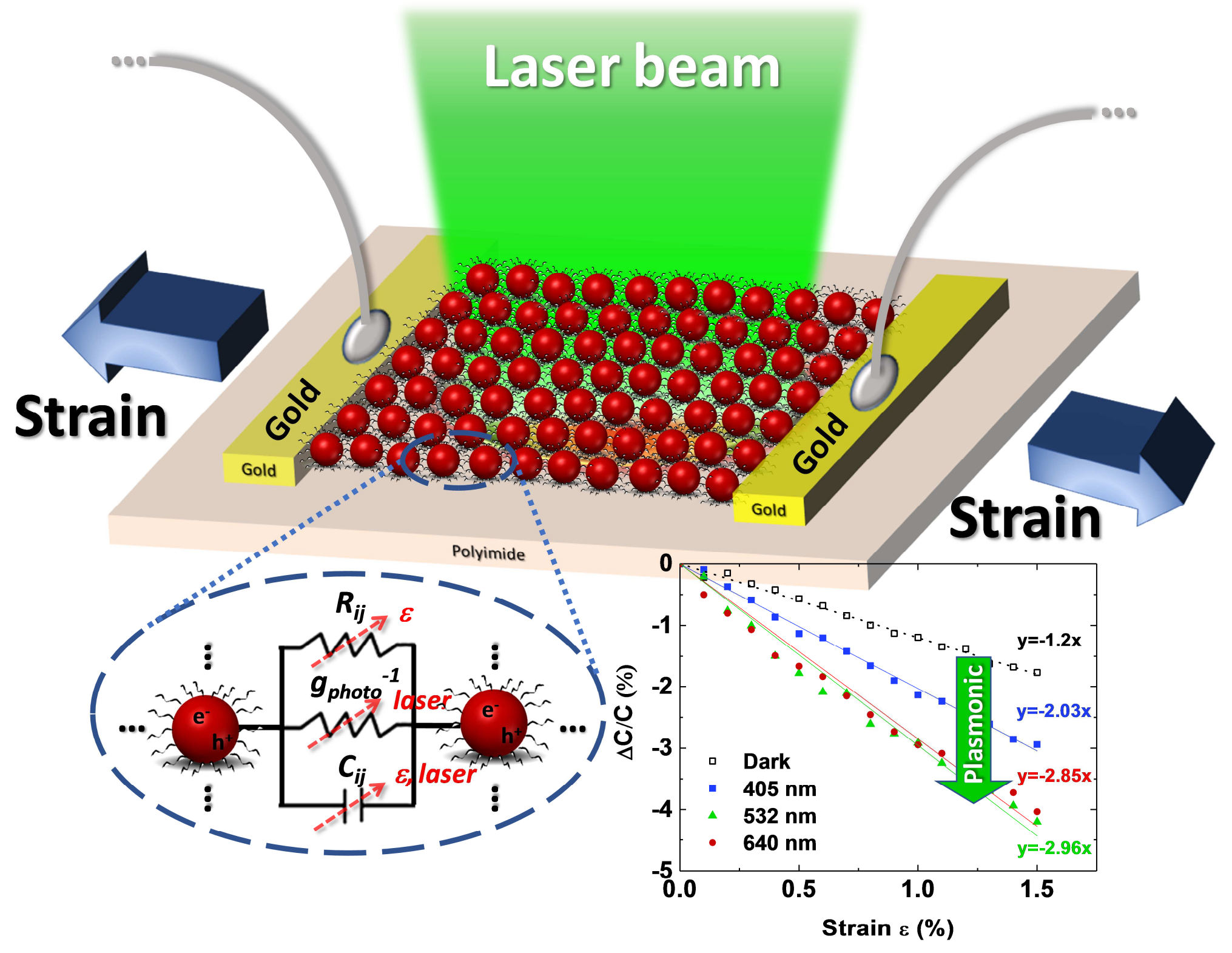

